\title{
How to use survey results
}

\author{
I. Diane Cooper, AHIP; Timothy P. Johnson, PhD
}

See end of article for authors' affiliations.

DOI: http://dx.doi.org/10.3163/1536-5050.104.2.016

Most library science surveys use Likert-style questions. After you read a study that reports results from Likert-style questions. After you read a study that reports results from Likert-style questions, you may think about using the results to guide some decision that you need to make. If so, then your job is to understand the validity of the results and how they apply to your decision. The researcher presents the results. You must determine how persuasive the results are for you.

\section{WHAT ARE LIKERT-LIKE QUESTIONS?}

You have probably filled out a questionnaire that asks you to choose one response from several that are offered. Commonly, the questions are in the Likert format. Likert-style questions involve responding to an assertion such as "I am satisfied with the new service," followed by a set of five possible responses. To understand and apply Likert and similar survey results, it is useful to be familiar with the art and science of these surveys and how you should approach their results.

In the most common Likert format, the middle response is usually one of the following: not sure, neither, neutral, or don't know; to the right of this midpoint are somewhat agree and strongly agree. To the left are a pair of responses that balance the set: somewhat disagree and strongly disagree. This is a five-point set of Likert response options, containing a middle point balanced by positive and negative options on either side. This is also known as a set of bipolar response options.

The possible response options are usually numbered 1 to 5 . For example, if the respondent selects strongly disagree, the datum for that question is " 1 " and so forth. The selected number is the data element used in statistical summaries of data collected. Likert questions can also have 7, 9, or more balanced response options, in which the neutral option would always occupy the midpoint of the response scale. But while it is essential that the response options are balanced, it is not required that a neutral midpoint be included [1].
Some survey questions are not in the balanced format; for example, they may only ask for "how much" something is used. These questions are called unipolar. Unipolar response options are frequently employed to measure quantities and frequencies, whereas bipolar formats are more commonly employed to measure attitudes and opinions. Sets of unipolar response options are often, and incorrectly, referred to as Likert measures.

\section{WHAT ARE STRENGTHS OF LIKERT-LIKE DATA?}

Because they are so widely used, researchers are comfortable constructing Likert items for their surveys. For the same reason, survey respondents are usually familiar with and comfortable responding to them. The style can be adapted to assess many dimensions of attitudes and opinions.

\section{WHAT ARE WEAKNESSES OF LIKERT-LIKE DATA?}

That middle spot, " 3 " in a 5-point scale, is often problematic, as many respondents - and researchers-are uncertain as to what it means. The survey respondent may be truly neutral, may not have enough information to make an informed choice (thus negating the value of the query), or may be trying to avoid giving a socially undesirable response ("I don't want to hurt anyone's feelings..."). On the other hand, eliminating the neutral "waffle" option may force respondents to endorse an answer that does not accurately reflect their opinions or lack thereof.

Questions must be carefully worded-and tested-so that a single narrow concept is described by each. One researcher wanted to know the service preferences of respondents. The researcher asked, "How much do you like or select to use (each service)?" Responses were (1) like very much, (2) like a little, (3) neither like nor dislike, (4) dislike a little, or (5) dislike very much. While the set of response options in this example are balanced, the question itself is ambiguous, having two distinct dimensions: 


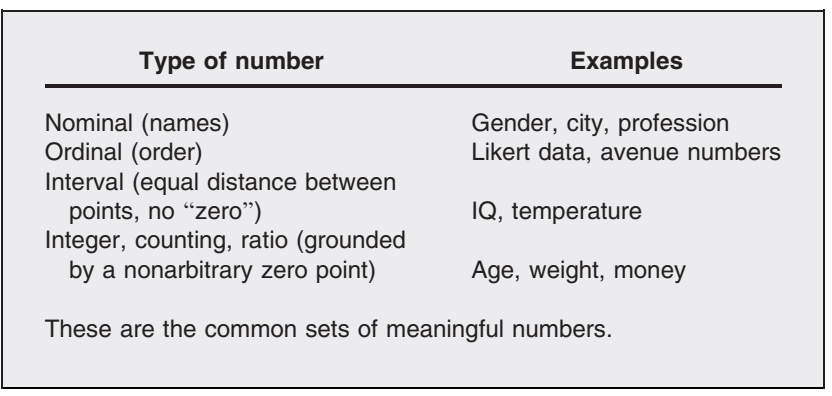

Table 1

Classifications of numbers

liking a service and selecting to use it are clearly different dimensions. In addition, the item asked about one end of the Likert options (liking) but not the other (disliking). Questions like these should not be persuasive for your decision making. A better strategy for collecting this information would have been to ask two separate questions: one that asked about like or dislike of the service, employing the existing set of Likert response options, and a second item that asked about the frequency of use of the service, employing a set of unipolar response options, perhaps: (1) always, (2) very often, (3) somewhat often, (4) not very often, (5) never.

Like all survey questions, the proper wording of Likert items can be very challenging in practice. What words should a researcher use to describe intensity of satisfaction, for example? Do the words mean the same to all populations, or are there age, cultural, or other confounders? If population subgroups respond differently, could it be only because they interpret the query differently, or are there differences in ability or motivation to recall information, form judgments, and/or report accurately? Sometimes finding more information about the subgroups that give different responses can reveal something else that affects their responses. Are they mostly very young? Is English their second language? These are called confounders.

\section{WHAT ARE THE PROBLEMS IN ANALYSIS AND PRESENTATION?}

\section{Ordinal data are used}

The elephant in the parlor of Likert response options, along with bipolar and unipolar survey questions in general, is that the sets of response options themselves are ordinal (Table 1).The numeric values commonly attached to the ordered response options in questionnaires serve multiple purposes. They organize data for analysis, but they also may communicate the perception that the response options are equidistant from one another (they are not). When coded as numbers for analysis, the values assigned to various response options take on a life of their own, as if respondents were selecting numbers when answering questions, rather than selecting one of the verbal response options provided.

The responses used for analysis are the numbers. There are many types of numbers. For research in general, the most common type of numbers are what we use to count, the counting numbers or integers or ratio numbers, which include negative numbers, zero, and positive (natural) numbers. These numbers have convenient characteristics: each interval between numbers is the same; the interval between two and nine is the same as the interval between three and ten. Data sets that fit this "equal interval" test are called parametric, a term originally used in geometry that has evolved to mean data for which it can be assumed that intervals are equal. Common statistical tests such as the $t$-test and analysis of variance (ANOVA) assume data have equal intervals (and that they are distributed in a nice bell-shaped Gaussian curve). Such tests are called parametric tests.

Ordinal values (such as Likert data) do not have this equal-interval characteristic. Rather, they are categorical labels that provide information regarding the ordering of some quality or construct, such as respondent satisfaction with a particular service. (Tip for recall: ordinal=order). Using parametric analyses is not appropriate for ordinal numbers. Every basic statistics reference source that we have found reiterates this. Studies that have ordinal results and use parametric analyses are subject to legitimate criticism on this point. Nursing research was recently criticized for this. A review of 166 nursing research papers found that only about half the researchers used appropriate nonparametric analyses [2]. There are numerous nonparametric tests that are more appropriate for use with survey items measured at ordinal and nominal levels of measurement. These are available in a variety of statistical software programs, including SAS, Stata, and SPSS.

\section{Distribution is important}

As mentioned above, the distance between Likert response options is not readily known and can vary across individuals. The location of small differences 
may be more important than the average or median value. For example, a 1-point separation around a neutral center response value of 3 and a somewhat dissatisfied value of 2, a separation of 1 point, can isolate respondents who are dissatisfied (2) from those who are not (3), which would seem to be an important finding, whereas a 1-point separation between response values of 4 and 5 only separates satisfied respondents into smaller groups, which may be of less importance. Under any circumstances, treating these distances as equal is probably not a good idea. Indeed, some available research has attempted to quantify respondent perceptions of intervals between survey response options, concluding that the assumption of equal distances between adjacent response options is not valid $[3,4]$. Consequently, using averages or even medians as adequate representation of the group may hide important information.

\section{Outliers are also a problem}

A few responses way off from the cluster of most responses can distort the results. Medians instead of means can be less sensitive to outlier distortion, and reporting a distribution's range can also provide useful information. But in many cases, the best analysis may be graphic. A simple histogram depicting the frequencies for each response can present otherwise hidden information.

It is important to distinguish Likert response options, such as those discussed above, from Likert scales, which are constructed additively from sets of questions employing Likert response options. Likert scales are not often used in health sciences librarian research. Although the response options to any given survey question should be treated as ordinal data, Likert scales will often have a much broader range of values, may approximate a normal distribution, and may be treated as interval or continuous data for purposes of statistical analysis [5].

\section{WHY DO RESEARCHERS USE PARAMETRIC ANALYSES ON ORDINAL DATA?}

In some cases, it is appropriate, as noted above when combining four or more Likert response questions to produce a Likert scale to describe a pattern or "personality."

Sometimes the researcher has enough data to demonstrate that the data have a normal distribution, and there is reasonable merit to assuming equal intervals.

And sometimes the study is a pilot study, not intended to be generalizable, and approximation is sufficient.

Keep in mind the possibility of confirmation bias. We are all human. Many researchers may have a subliminal desire to prove their hypotheses are correct. Parametric analyses are "more powerful"; in other words, they can find a statistical difference that is not identified by nonparametric analyses. And researchers are aware of a negative publication bias: studies that do not find a significant difference are less likely to be published [6]. So they may have subtle, sometimes unconscious, incentives to find "significance" and go with the analytic procedure most likely to declare it, which may be parametric.

\section{WHAT YOU, THE READER, SHOULD DO CONSIDERING SURVEY DATA}

1. First, look at the sample. Who were the respondents who completed the questionnaire or who participated in in-person or telephone interviews? If the sample is not representative-cannot claim to reflect a balanced view of the population of interest-be very careful in allowing the results to persuade you.

2. Then, look carefully at the key questions. Are they worded in a way that is clear and relevant to your interests? Do they really capture the construct proposed by the researcher?

3. Then, look at the analyses. If the data are single items employing Likert response options, the analysis should be nonparametric (not $t$-tests or ANOVAs), or the authors should provide a reasonable explanation of why it is not nonparametric.

4. Then, look at the results. Consider how many respondents did not really offer a definitive opinion, in other words, a " 3 " on a 1-to-5 balanced scale. A nice histogram may be the best way to consider the data. 5. Look to see if the authors have acknowledged and addressed any limitations to their measures or analyses that you have identified.

Use your observations on these points to determine how persuasive the study is for your needs.

\section{REFERENCES}

1. Colton D, Covert RW. Designing and constructing instruments for social research and evaluation. San Francisco, CA: Jossey-Bass; 2007. 
2. Jakobsson U. Statistical presentation and analysis of ordinal data in nursing research. Scand J Caring Sci. 2004; 18:437-40.

3. Hougland JG, Johnson TP, Wolf JG. A fairly common ambiguity: comparing rating and approval measures in public opinion polling. Sociol Focus. 1992;25(3):257-71. 4. Johnson T, O’Rourke D, Chavez N, Sudman S, Warnecke R, Lacey L, Horm J. Social cognition and responses to survey questions among culturally diverse populations. In: Lyberg L, Biemer P, Collins M, de Leeuw E, Dippo C, Schwarz N, Trewin D, eds. Survey measurement and process quality. New York, NY: John Wiley \& Sons; 1996. p. 87-113. 5. Carifio J, Perla RJ. Ten common misunderstandings, misconceptions, persistent myths and urban legends about Likert scales and Likert response formats and their antidotes. J Soc Sci. 2007;3(3):106-16.
6. Dickersin K. The existence of publication bias and risk factors for its occurrence. JAMA. 1990 Mar 9;263(10): 1385-99.

\section{AUTHORS' AFFILIATIONS}

I. Diane Cooper, AHIP, jmlaeditorbox@gmail.com, Editor-in-Chief, Journal of the Medical Library Association; Timothy P. Johnson, PhD, timj@uic.edu, Director, Survey Research Laboratory and Professor, Public Administration, University of Illinois at Chicago, 1240 Harrison Street, Suite 1595, Chicago, IL 60607 